Original

\title{
Prevalencia de hipovitaminosis $D$ en población trabajadora sanitaria
}

\author{
Prevalence of hypovitaminosis D in population Health Worker
}

\author{
Brígido Pérez Bermúdez ', M. Antonio Aranda Vizcaíno ', Javier Rodríguez de Cía ${ }^{2}$, Carolina Corvalán Prano ${ }^{3}$ \\ Carmen Fernández Felipe ${ }^{3}$
}

1. Servicio de Prevención de Riesgos Laborales. Hospital Universitario de La Princesa. Madrid. España.

2. Hospital Universitario "Sta. Cristina". Madrid. España.

3. Unidad Docente. Hospital Universitario de La Princesa. Madrid. España.

Recibido: 16-10-12

Aceptado: 28-11-12

\section{Correspondencia}

Brígido Pérez Bermúdez

Servicio de Prevención de Riesgos Laborales

Hospital Universitario de La Princesa. Área 2

Diego de León, 62

28066 Madrid. España

Tfno: 915202569

Correo electrónico: bperezb.hlpr@salud.madrid.org

Resumen

Introducción: En los últimos años han ido apareciendo un gran número de artículos científicos evidenciando la elevada prevalencia de hipovitaminosis D en la población general.

Objetivos: Analizar los niveles de vitamina D (vit. D) en un colectivo de trabajadores sanitarios.

Material y métodos: Estudio descriptivo. Se analizaron mediante un inmunoensayo quimioluminiscente de micropartículas (CLIA), el analito $25 \mathrm{OH}$ vit. D, y la Parathormona (PTH). Para el análisis bivariante se aplicó una chi cuadrado y la t-Student. La significación se consideró $\mathrm{p}<0.05$. Posteriormente se realizó una Regresión Logística (R.L.), cuya medida de asociación fue la Odds Ratio (O.R). Se han construido dos modelos uno considerando déficit de vit. D niveles inferiores a $30 \mathrm{ng} / \mathrm{ml}$, y otro para niveles inferiores a $20 \mathrm{ng} / \mathrm{ml}$.

Resultados: Se ha observado una prevalencia de hipoavitaminosis D de $84.9 \%(80.8 \%-89.1 \%)$. Las variables tomar el Sol o UVA en el último mes, junto a los niveles de PTH, son las variables que están relacionadas con la Hipovitaminosis. Curiosamente, cuando se construye el modelo de R.L. con corte a los $20 \mathrm{ng} / \mathrm{ml}$ la única variable significativa es la exposición al sol.

Conclusiones: Existe una prevalencia muy alta de hipovitaminosis D en los trabajadores sanitarios estudiados. La PTH tiene un comportamiento singular en función del punto de corte considerado en la vit.D.

Med Segur Trab (Internet) 2012; 58 (229) 335-344

Palabras clave: Deficiencia vitamina D, Paratiroides y Vitamina D, Trabajadores sanitarios. 


\section{Abstract}

Introduction: In recent years have appeared a large number of scientific evidence of the high prevalence of hypovitaminosis $\mathrm{D}$ in the general population.

Objectives: To analyze the levels of vit. D in a group of health workers.

Methods: Descriptive study. Were analyzed by through microparticles chemiluminescent immunoassay (CLIA), the analyte $25 \mathrm{OH}$ vit. D and Parathormone (PTHi). For the bivariate analysis chi-square an Student's $\mathrm{t}$ test was applied. Significance was considered $\mathrm{p}<0.05$. Subsequently, it was performed a logistic regression (L.R.) whose measure of association was the odds ratio (OR). It has built two models, one considering deficit vit. D levels below $30 \mathrm{ng} / \mathrm{ml}$, and another to levels, below $20 \mathrm{ng} / \mathrm{ml}$.

Results: It has been observed a prevalence of hipoavitaminosis D, of $84.9 \%(80.8 \%-89.1 \%)$. Variables sunbathing or UVA in the last month together with PTHi levels, are the variables that are associated with hypovitaminosis Interestingly, when constructing LR model will cut at $20 \mathrm{ng} / \mathrm{ml}$, sun exposure is the only significant variable.

Conclusions: There is a very high prevalence of hypovitaminosis D in the health worker population studied. PTH has an odd behavior according with vit. D cutoff considered.

Med Segur Trab (Internet) 2012; 58 (229) 335-344

Key words: Vitamin D Deficiency, Parathyroid hormone and Vitamin D, health workers. 


\section{INTRODUCCIÓN}

En los últimos años han ido apareciendo un gran número de artículos científicos evidenciado las elevadas prevalencias de hipovitaminosis D en la población general ${ }^{1-4}$. Como sabemos, esta vitamina, considerada como liposoluble, es en realidad una hormona esteroidea que está involucrada en la regulación del complejo sistema de la homeostasis mineral manteniendo específicamente la integridad del hueso y estando también implicada en la diferenciación celular en una gran diversidad de tejidos. En el hombre, el $90 \%$ de la vitamina $\mathrm{D}$ se aporta por la exposición al sol. En la epidermis la radiación solar transforma el 7-deshidrocolesterol en colecalciferol mediante la acción de los rayos ultravioleta B. El $10 \%$ restante se obtiene directamente de los alimentos, especialmente de los aceites de pescado. Posteriormente e independientemente del origen, el colecalciferol sufre una primera hidroxilación en el hígado, formando la $25 \mathrm{OHD}_{3}$, aceptándose éste como el mejor metabolito indicativo del estatus de Vitamina D por su mayor vida media, aproximadamente tres semanas. Posteriormente, mediante una nueva hidroxilación a nivel renal, se sintetiza el calcitriol $\left(1,25(\mathrm{OH})_{2} \mathrm{D}\right)$, siendo ésta la molécula biológicamente activa. La Vitamina D, junto con la Parathormona (PTH), está estrechamente relacionada con el metabolismo del calcio y del fósforo, consiguiendo entre estos cuatro actores el equilibrio necesario para la salud básica del hueso ${ }^{5}$. Entre otras funciones, también está implicada en la regulación, maduración y crecimiento celular, inhibe la producción de renina, incrementa la secreción de insulina, y actúa sobre los linfocitos B y T. Se calcula que estas hormonas y oligoelementos regulan la transcripción de aproximadamente un 3\% del genoma humano.

No existe un consenso para determinar los valores óptimos de $25(\mathrm{OH})$ vitamina $\mathrm{D}$. Se acepta que con valores inferiores a $9.9 \mathrm{ng} / \mathrm{mL}$ estaríamos en deficiencia, entre 10 y $19.9 \mathrm{ng} / \mathrm{mL}$ estaríamos hablando de insuficiencia, entre 20 y $29.9 \mathrm{ng} / \mathrm{mL}$ seria un déficit relativo. Es a partir de $30 \mathrm{ng} / \mathrm{mL}$ cuando se consideran cifras normales, aunque parece que los últimos consensos resuelven que para mantener una buena salud ósea los niveles no deben de ser inferior a $20 \mathrm{ng} / \mathrm{mL}^{6,7}$.

Existen factores que pueden determinar que existan bajos niveles de vitamina $\mathrm{D}$, como son la edad, las horas de exposición solar, la estación del año, el fototipo del individuo (capacidad de la piel para asimilar la radiación solar), el uso de cremas protectoras en piel, la latitud del lugar, enfermedades asociadas con malabsorción de grasas, hepáticas y renales ${ }^{8,9}$, etc.

Por otra parte existen trabajos que no solo relacionan el déficit de vitamina $\mathrm{D}$ con alteraciones en el metabolismo del Calcio y fósforo (Osteoporosis) y aumento de fracturas, sino que han relacionado deficiencias de vitamina $\mathrm{D}$ con enfermedades cardiovasculares, alteraciones cognitivas, cáncer mama, próstata, colon y enfermedades autoinmunes y con un aumento de la mortalidad en general ${ }^{10-16}$.

Nosotros desde nuestra área de Salud Laboral hemos realizado este trabajo con el fin de analizar los niveles de vitamina $\mathrm{D}$ en un colectivo de trabajadores sanitarios.

\section{MATERIAL Y MÉTODOS}

Estudio descriptivo realizado entre octubre de 2010 y octubre de 2011, sobre una población de 306 sanitarios del Hospital Universitario de Santa Cristina y del Hospital Infantil Universitario Niño Jesús de Madrid.

La determinación de la Vitamina $\mathrm{D}(25 \mathrm{OH})$ se realizó por un inmunoensayo quimioluminiscente de micropartículas (CMIA), al igual que la PTH intacta. Calcio y fósforo se midieron por técnicas espectrofotométricas. Los valores de referencia aplicados a la población estudiada fueron los siguientes: Vitamina D: estado de suficiencia $>30 \mathrm{ng} /$ ml, PTHi: 10-65 pg/ml, Calcio: 8.4-10.2 mg/dl y Fósforo: 2.3-4.7 mg/dl. 
Los sujetos estudiados fueron trabajadores sanitarios que acudían a los exámenes de salud y que voluntariamente aceptaban realizar una encuesta en la que se recogía la edad, sexo, categoría laboral, índice de masa corporal (IMC), si estaba en periodo de gestación, padecimiento de patologías, medicación, hábito tabaquico, si realizaba nocturnidad, ejercicio al aire libre, dieta pobre en grasa, tratamiento con estatinas y si había tomado el sol o rayos ultravioletas en el último mes, estación anual en la que se obtiene la muestra y los niveles de Calcio, Fósforo y Parathormona (PTH). Respecto a la inclusión de la exposición de los rayos ultravioletas A, se consideró que si bien es verdad que los rayos ultravioletas que producen la conversión de 7-dehidrocolesterol a previtamina D3 son los rayos UVB (longitud de onda entre 290 y $315 \mathrm{~nm}$ ) la exposición a las lámparas UVA (320$400 \mathrm{~nm}$ ) por razones estéticas entre la población femenina es muy frecuente y este tipo de lámparas también producen emisiones de rayos UVB, más si sus mantenimientos no son eficientes.

Para el análisis bivariante de proporciones se aplicó una chi cuadrado y para variables continuas se aplicó la t-Student. La significación se consideró cuando p< 0,05.

Posteriormente se utilizó un análisis multivariante (Regresión Logística) cuya medida de asociación fue la Odds Ratio (O.R) y se calcularon sus Intervalos de Confianza (I.C.) al $95 \%$. Se han realizado dos modelos de Regresión Logística, un modelo considerando déficit de vitamina $\mathrm{D}$ niveles inferiores de $30 \mathrm{ng} / \mathrm{mL}$, y otro considerando el déficit en los niveles inferiores a $20 \mathrm{ng} / \mathrm{mL}$.

\section{RESULTADOS}

En nuestro estudio se ha observado una prevalencia de hipoavitaminosis $\mathrm{D}$, de 84.9\% (80.8\% - 89.1\%), es decir solo un $15 \%$ de los trabajadores estudiados tenían valores considerados normales ( > a $30 \mathrm{ng} / \mathrm{mL}$ ). Las prevalencias para los distintos niveles se muestran en la Tabla I.

Tabla I. Resultado de los niveles de vitamina D

\begin{tabular}{lccc}
\hline \multicolumn{1}{c}{ Niveles de Vita. D } & FRECUENCIA & $\%$ & I.C al 95 \% \\
\hline$>30 \mathrm{ngr} / \mathrm{mL}$ & 46 & 15.03 & $(10.86-19.20)$ \\
De 20 a $29.9 \mathrm{ngr} / \mathrm{mL}$ & 105 & 34.31 & $(28.83-39.79)$ \\
De $10 \mathrm{a} 19.9 \mathrm{ngr} / \mathrm{mL}$ & 132 & 43.13 & $(37.42-48.85)$ \\
$<9.9 \mathrm{ngr} / \mathrm{mL}$ & 23 & 7.51 & $(4.39-10.63)$ \\
\hline
\end{tabular}

Los resultados del descriptivo y el bivariante se observan en la Tabla II.

Tabla II. Descriptivo y bivariante

\begin{tabular}{lrrr}
\hline \multicolumn{1}{c}{ Variables } & $>30 \mathrm{ng} / \mathrm{mL}$ & $<29.9 \mathrm{ng} / \mathrm{mL}$ & $\mathrm{p}$ \\
\hline Mujer & $40(14.7 \%)$ & $232(85.3 \%)$ & \\
Hombre & $6(17.6 \%)$ & $28(82.4 \%)$ & $0,651^{*}$ \\
\hline Sanitarios (Médicos-Enfermeros) & $16(12.7 \%)$ & $110(87.3 \%)$ & \\
No Sanitarios & $30(16.7 \%)$ & $150(83.3 \%)$ & $0.339^{*}$ \\
\hline No Nocturnidad & $29(17.4 \%)$ & $138(82.6 \%)$ & \\
Nocturnidad & $17(12.2 \%)$ & $122(87.8 \%)$ & $0.137^{*}$ \\
\hline No fumador o menos de 5 cigarrillos & $41(16.4 \%)$ & $209(83.6 \%)$ & \\
Fumador de mas de 5 cigarrillos & $5(9.4 \%)$ & $48(90.6 \%)$ & $0.199^{*}$ \\
\hline
\end{tabular}




\begin{tabular}{|c|c|c|c|}
\hline Variables & $>30 \mathrm{ng} / \mathrm{mL}$ & $<29.9 \mathrm{ng} / \mathrm{mL}$ & $\mathbf{p}$ \\
\hline Tomar Sol o UVAs último mes & $27(24.5 \%)$ & $83(75.5 \%)$ & \\
\hline No tomar Sol o UVAs íltimo mes & $19(9.8 \%)$ & $175(90.2 \%)$ & 0.001* \\
\hline Meses de Noviembre a Abril & $9(7.8 \%)$ & $107(92.2 \%)$ & \\
\hline Meses de Mayo a Octubre & $37(19.5 \%)$ & $153(80.5 \%)$ & $0.005^{*}$ \\
\hline No Ejercicio (< de $30 \mathrm{~min} . /$ día) & $23(13.2 \%)$ & $151(86.8 \%)$ & \\
\hline Si Ejercicio (> de 30 min./día) & $23(17.7 \%)$ & $107(82.3 \%)$ & $0.282^{*}$ \\
\hline Dieta sin grasas & $10(14.7 \%)$ & $58(85.3 \%)$ & \\
\hline Dieta con grasas & $36(15.1 \%)$ & $202(84.9 \%)$ & $0.932 *$ \\
\hline Toma Estatinas & $3(15.0 \%)$ & $17(85.0 \%)$ & \\
\hline No toma Estatinas & $43(15.0 \%)$ & $243(85.0 \%)$ & 1.000 \\
\hline Edad (años) & $45.4(11.6)^{* * *}$ & $46.5(11.7) * *$ & $0.563^{* * * *}$ \\
\hline IMC & $24.2(3.6) * *$ & $25.2(4.5) * *$ & $0.155^{\text {**** }}$ \\
\hline $\mathrm{Ca}^{++} \mathrm{ng} / \mathrm{ml}$ & $9.9(4.4) * *$ & $9.1(0.4) * *$ & $0.284^{* * *}$ \\
\hline $\mathrm{P}^{++} \mathrm{ng} / \mathrm{ml}$ & $3.6(0.5) * *$ & $3.6(0.8) * *$ & $0.898^{* * * *}$ \\
\hline PTH pg/ml & $44.1(17.5) * *$ & $52.0(21.3) * *$ & $0.028^{* * * *}$ \\
\hline
\end{tabular}

$\left({ }^{*}\right)=\chi^{2}$

$\left({ }^{* *}\right)=$ Media (desviación típica)

$(* * *)=t-$ Student

Como puede verse las variables tomar el Sol o UVA en el último mes junto, la variación estacional de verano e invierno y los niveles de Parathormona, son las variables que están relacionadas con la Hipovitaminosis $\mathrm{D}$, de tal forma que durante los meses de verano y la toma de sol/uva en el último mes hace que la prevalencia de hipovitaminosis sea menor y por otra parte los niveles de PTH aumentan cuando los niveles de vitamina D descienden. El resto de las variables analizadas no han sido significativas.

La Tabla III resume el resultado del modelo de Regresión Logística, en el que se puede observar que las variables que son significativas son la exposición al sol o tomar rayos UVA en el ultimo mes (O.R=0.389) es decir existe 2.6 veces menos de Odds (1/0.38) de no padecer hipovitaminosis $\mathrm{D}$, si se ha tomado el Sol /UVA en el último mes. Por otra parte por cada $1 \mathrm{ng} / \mathrm{mL}$ que disminuye la Vitamina D en suero, aumenta la PTH un $2 \%$. El resto de las variables no son significativas, incluso la variable estacional (veranoInvierno) que sí lo era en el bivariante, deja de serlo como parece lógico. Lo que significativamente está relacionado con no padecer hipovitaminosis D es estar más expuesto al Sol y generalmente en nuestra latitud y cultura es en verano cuando más lo estamos. Al modelo se le comprobó la bondad de ajuste mediante el test de Hosmer y Lemeshow, resultando una $\mathrm{p}=0.902$ y un área de la curva ROC de 0,79 a los valores de las probabilidades calculadas mediante el modelo.

TABLA III. Regresión Logística para punto de corte $30 \mathrm{ng} / \mathrm{ml}$

\begin{tabular}{lccc}
\hline \multicolumn{1}{c}{ Variables } & $\mathbf{p}$ & O. R. & I. C. al 95 \% \\
\hline Sexo & 0.842 & 0.891 & $(0.286-2.780)$ \\
Edad & 0.950 & 0.999 & $(0.962-1.037)$ \\
Sanitarios & 0.479 & 0.713 & $(0.279-1.822)$ \\
Nocturnidad & 0.475 & 1.396 & $(0.559-3.482)$ \\
Fumador & 0.495 & 1.504 & $(0.465-4.859)$ \\
Ejercicio & 0.464 & 0.750 & $(0.357-1.572)$
\end{tabular}




\begin{tabular}{|c|c|c|c|}
\hline Variables & $\mathbf{p}$ & O. R. & I. C. al $95 \%$ \\
\hline Sol / U.V.A Último mes & 0.016 & 0.389 & $(0.180-0.839)$ \\
\hline Verano / Invierno & 0.127 & 2.016 & $(0.820-4.956)$ \\
\hline Estatinas & 0.721 & 1.345 & $(0.264-6.851)$ \\
\hline Dieta sin grasas & 0.974 & 1.015 & $(0.410-2.515)$ \\
\hline IMC & 0.239 & 1.067 & $(0.958-1.188)$ \\
\hline $\mathrm{Ca}^{++}$ & 0.557 & 0.801 & $(0.381-1.682)$ \\
\hline $\mathrm{P}^{++}$ & 0.515 & 1.199 & $(0.694-2.071)$ \\
\hline PTH & 0.049 & 1.021 & $(1.001-1.042)$ \\
\hline
\end{tabular}

La Tabla IV muestra los resultados de la Regresión Logística con punto de corte a los $20 \mathrm{ng} / \mathbf{m L}$. Como se puede observar, la significación de la PTH, ha desaparecido y la única variable que persiste siendo significativa es la exposición al sol, con una O.R. de 0. 43. Es decir, existe un efecto protector frente a la hipovitaminosis $\mathrm{D}$ al tomar el sol. Los trabajadores que se han expuesto a la radiación solar en el último mes tienen 2.3 veces menos riesgo (1/0.43) de tener niveles bajos de vitamina $D$. El cálculo de la O.R. respecto a la toma de sol es similar si se considera el punto de corte $20 \mathbf{n g} / \mathbf{m L}$ o $30 \mathbf{n g} / \mathbf{m L}$. En resumen, el que se expone al sol tiene 2,5 veces más O.R. de no padecer hipoavitaminosis D.

Tabla IV. Regresión Logística para punto de corte $20 \mathrm{ng} / \mathrm{mL}$

\begin{tabular}{lccc}
\hline \multicolumn{1}{c}{ Variables } & $\mathbf{p}$ & $\mathbf{O} . \mathbf{R}$. & I. C. al $\mathbf{9 5} \%$ \\
\hline Sexo & 0.220 & 0.583 & $(0.246-1.381)$ \\
Edad & 0.886 & 0.998 & $(0.973-1.024)$ \\
Sanitarios & 0.456 & 1.256 & $(0.690-2.288)$ \\
Nocturnidad & 0.134 & 0.628 & $(0.341-1.155)$ \\
Fumador & 0.556 & 1.232 & $(0.616-2.465)$ \\
Ejercicio & 0.528 & 1.187 & $(0.697-2.021)$ \\
Sol / U.V.A Último mes & $\mathbf{0 . 0 0 5}$ & $\mathbf{0 . 4 3 7}$ & $\mathbf{( 0 . 2 4 4}-\mathbf{0 . 7 8 2})$ \\
Verano / Invierno & 0.140 & 1.525 & $(0.871-2.671)$ \\
Estatinas & 0.364 & 1.652 & $(0.558-4.890)$ \\
Dieta sin grasas & 0.783 & 1.097 & $(0.569-2.112)$ \\
IMC & 0.382 & 1.027 & $(0.967-1.091)$ \\
Ca & ++ & 0.921 & $(0.699-1.213)$ \\
$\mathrm{P}^{++}$ & 0.556 & 0.824 & $(0.575-1.181)$ \\
PTH & 0.291 & 3.977 & $(0.351-1.790)$ \\
\hline
\end{tabular}

La figura 1 representa mediante un gráfico de dispersión el comportamiento de ambas variables, PTH y Vitamina D. Como puede observarse en el corte a $30 \mathbf{n g} / \mathbf{m L}$ se observan que casi todos los niveles, excepto tres registros, de PTH están dentro de lo que se consideran limites normales $(10-65 \mathrm{pg} / \mathrm{ml})$ ). Pero cuando el corte de la Vitamina D lo situamos en $20 \mathrm{ng} / \mathrm{mL}$, los registros con niveles de PTH elevados parecen distribuirse a ambos lados del corte. 
Gráfico 1. Gráfico de Dispersión Vitamina D - PTH

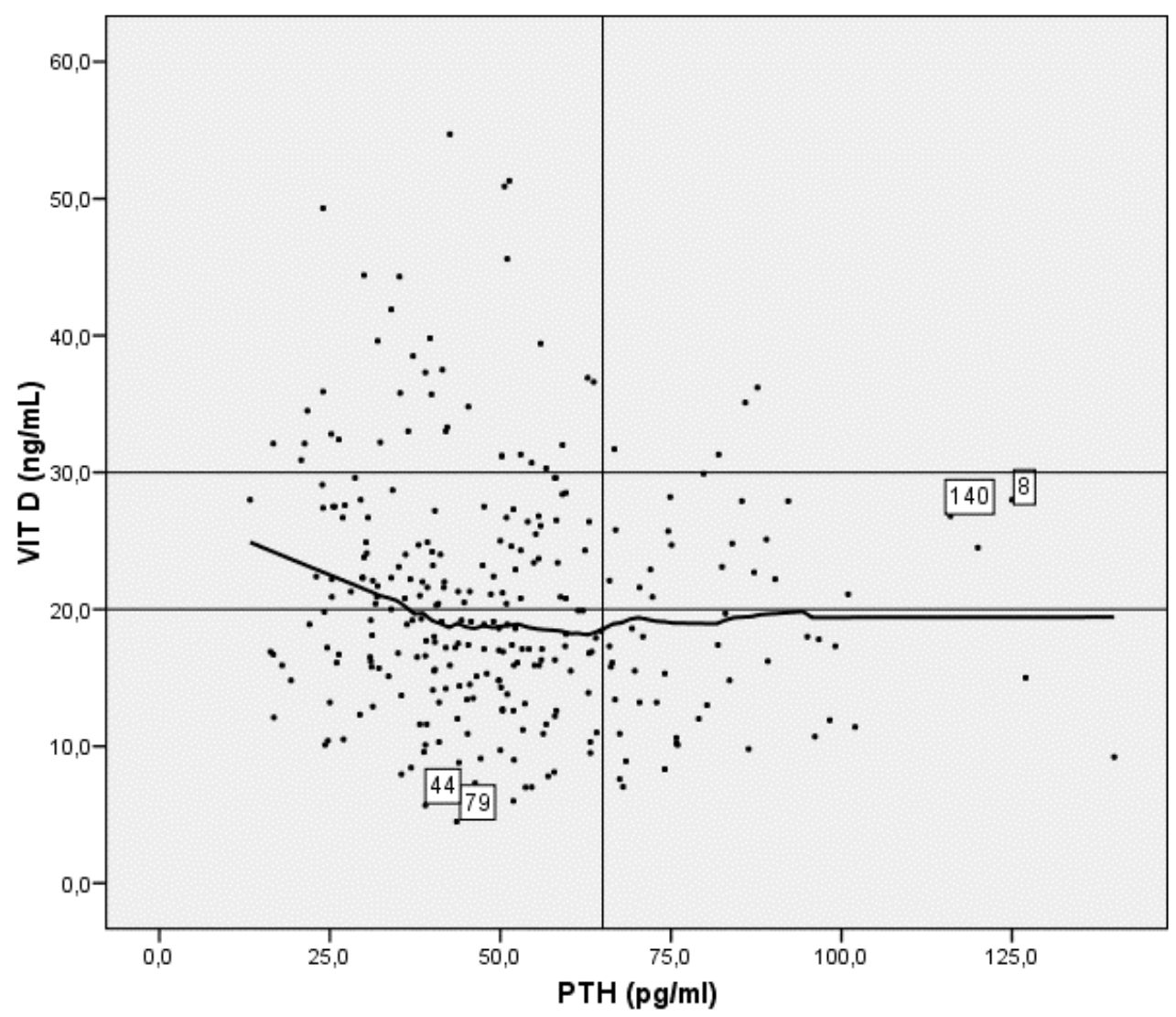

\section{DISCUSIÓN}

Este estudio se ha diseñado tras tener constancia de numerosos estudios en los que se ha visto la elevada prevalencia de hipovitaminosis D que existe en la población general. Nos preguntábamos si este déficit se confirmaría en una población relacionada con la sanidad y si las características de su trabajo (categorías profesional, turnos) y hábitos (fumador, ejercicio, dietas, etc.) podrían favorecer o proteger de esta deficiencia. En los resultados obtenemos como primera evidencia, que este déficit de $25 \mathrm{OHD}_{3}$ es parecido a la de otros estudios realizados sobre población general, no influyendo la profesión de sanitaria en la prevalencia de esta alteración.

Como segunda conclusión llama la atención que solamente la exposición solar está relacionada significativamente, su efecto es protector frente a este déficit de vitamina $D$. En la literatura española revisada, todos los investigadores se sorprenden del hallazgo que en un país como el nuestro, con elevadas horas de insolación, la población presente prevalencias de hipovitaminosis D semejante a la de otros países con latitudes más al norte, incluso prevalencias más altas ${ }^{(17)}$. Se alude como explicación que a pesar de la latitud en la que nos hayamos, nos exponemos en realidad poco al sol. Por una parte porque España está por encima del paralelo $35^{\circ}$ y la exposición al sol en invierno no es efectiva para la síntesis de vitamina $\mathrm{D}$, siendo necesario que esta exposición se realice en las horas centrales del día y por otra, en verano, debido a la excesiva temperatura (en esas horas), tratamos de evitar la exposición directa al sol. Si a esto sumamos que cuando tenemos la intención de tomar el sol, se aconseja de forma reiterada y a instancias de las autoridades y profesionales sanitarios, el uso de cremas protectoras con elevados factores de protección con el fin de evitar el riesgo del cáncer de piel, tendríamos las razones para explicar que a pesar de nuestra latitud la exposición efectiva al sol es baja. 
Por ultimo, respecto a las consideraciones sobre la exposición al sol y en concreto en la muestra analizada, se podría argumentar que dado que estas cifras de hipovitaminosis D pertenecen a una población urbana y trabajadora, con el consiguiente ritmo de vida que se genera en las grandes ciudades, y sumando que este tipo de trabajadores están sometidos a turnicidad laboral, sería posible jujtificar, en base a ello, las cifras de vitamina D encontradas en la misma. Pero como vemos en nuestro estudio, la variable turnicidad no es significativa y en otros estudios ${ }^{2,4,5}$, se confirman igualmente estas prevalencias en otras poblaciones menos urbanas.

Sabemos que la Vitamina $D$ es una vitamina liposoluble y que alimentos como, (aparte del famoso aceite hígado de bacalao que no se ingiere habitualmente en la población general), el salmón, el atún, las sardinas y la caballa entran frecuentemente en la dieta mediterránea y poseen abundante contenido de esta vitamina. Sin embargo, parece demostrarse que nuestra ingesta, aproximadamente $200 \mathrm{UI} /$ día, es inferior a la encontrada en los países escandinavos, alrededor de $400 \mathrm{UI} /$ día $^{(18)}$ de media. Por otra parte, en comparación con otros países situados más al norte, nosotros tenemos pocos alimentos enriquecidos con vitamina $\mathrm{D}$, y por tanto la ingesta exógena sería poco eficiente para proporcionar las necesidades del organismo. En nuestro análisis recogimos una variable en la que preguntábamos al trabajador si éste realizaba en los últimos tres meses dieta pobre en grasas, no obteniendo como observamos significación entre la hipovitaminosis D y el realizar dieta baja en grasas. También contemplamos la idea de recoger el consumo de estatinas por la misma razón, ya que al ser una vitamina liposuble podrían verse afectados sus niveles por una alteración en el metabolismo de los lípidos, no encontrando tampoco diferencias significativas.

En relación con lo anterior, es decir, con posibles factores que puedan influir en el aporte exógeno de Vitamina $\mathrm{D}$, que los turnos rotatorios con la nocturnidad, no solo podría influir en la menor exposición solar sino que al producir alteraciones en los biorritmos repercutiría también, entre otras facetas, en la alimentación, afectando a la cantidad de ingesta alimentaria sino también a su calidad, siendo más proclive el trabajador a una alimentación de comidas rápidas (bocadillos, sándwiches, pizzas, etc.) y alimentos de repostería (bollería, dulces, etc.), con menos riqueza en vitaminas. Pero como ya hemos mencionado, esta variable no es significativa respecto a otros trabajadores con turnos de trabajo más convencionales.

Una consideración aparte merece las observaciones realizadas respecto a la PTH, comprobándose un comportamiento peculiar. Situando el punto de corte en $30 \mathbf{n g} / \mathbf{m L}$, se observa que la relación con la PTH es inversa, es decir a medida que disminuye la Vitamina D aumenta la PTH y esta correlación es significativa. Si el punto de corte lo situamos en $20 \mathrm{ng} / \mathbf{m L}$ se observa que la significación encontrada desaparece, a un lado y a otro de este dintel existen igualmente registros con PTH elevadas. Vemos al analizar registros de manera individual que existen determinaciones con vitamina D bajas (Ejemplo registros número 44 y 79 del (gráfico I) que no necesariamente se corresponden con elevaciones de PTH y viceversa registros con elevadas PTH (registros n. 8 y 140) que no se corresponden con niveles bajos de vitamina D. En los estudios publicados hasta ahora no hemos encontrado ninguna referencia a esta singularidad. Es posible que algunos trabajadores estudiados tengan niveles elevados de PTH por alteración de las Paratiroides y no por la deficiencia de Vitamina $\mathrm{D}$, creando algún efecto confusor no controlado. $\mathrm{O}$ el origen del hiperparatiroidismo podría hallarse en una alteración en la síntesis renal de $1,25(\mathrm{OH})_{2}$ vit.D.

Es muy llamativo, que sea cual sea la causa de estas alteraciones en los niveles de Vitamina D y de la PTH, las concentraciones de los iones calcio y fósforo que hemos observado en nuestro estudio son siempre normales. Aunque es muy conocido y estudiado este complejo y dinámico equilibrio donde lo fundamental es precisamente mantener estos niveles de los iones en sus valores normales. Debemos de concluir que para que estos electrolitos se vean alterados es necesario unos niveles aún mucho más deficitarios en la vitamina $\mathrm{D}$, al igual que ocurre en todos los estudios revisados los iones no están alterados en sangre. Ante todo lo expuesto, cabe preguntarse si las cifras que manejamos 
como valores de referencia para clasificar lo que consideramos cifras normales o hipovitaminosis son las apropiadas, estamos manejando el dintel de $30 \mathrm{ng} / \mathrm{mL}$ en los laboratorios pero ya algunos consensos los sitúan por debajo de $20 \mathrm{ng} / \mathrm{mL}^{(19)}$. Aun admitiendo este dintel, las cifras de hipovitaminosis D serían altas, en nuestro estudio de un 50.6\%. Tras el análisis de los datos y las nulas repercusiones clínicas de estas prevalencia de hipovitaminosis $\mathrm{D}$, así como la nula repercusión en los niveles de Calcio y Fósforo en suero, cabría contemplar otras preguntas ¿es el 25-OH-D3 (Calcifediol) un buen indicador para definir la hipovitaminosis D?, ¿Podría existir un mediador aún no estudiado y definido que regule la conversión de 25-OH-D3 (Calcifediol) a 1,25(OH)2D (Calcitriol) que es la molécula activa? o ¿mediante otra vía producir Calcitriol sin ser necesario el precursor Calcifediol?

Finalmente pensamos que si aceptamos que la determinación de la $25 \mathrm{OHD}_{3}$ es un buen parámetro para la determinación de la Vitamina $\mathrm{D}$ en el individuo, se debería de considerar la posibilidad de recomendar a la población la conveniencia de exponerse al sol de forma moderada, especialmente en ancianos y niños, al contrario de los mensajes taxativos de que dicha exposición es dañina por el potencial riesgo de cáncer de piel. También sería conveniente reflexionar sobre la conveniencia de enriquecer alimentos con vitamina D y más en poblaciones urbanas y del norte donde este aporte puede ser un suplemento importante para disminuir la prevalencia de este déficit de Vitamina D.

\section{REFERENCIAS BIBLIOGRÁFICAS}

1. González-Padilla E, Soria López A, González-Rodríguez E, García-Santana S, Mirallave-Pescador A, Groba Marco M, et al. Elevada prevalencia de hipovitaminosis D en los estudiantes de medicina de Gran Canaria, Islas Canarias (España). Endocrinol Nutr. 2011;58(6):267-73.

2. Vaqueiro M, Baré M, Anton A, Andreu E, Moya A, Sampere R, et al. Hipovitaminosis D asociada a exposición solar insuficiente en la población mayor de 64 años. Med Clin (Barc). 2007;129(8):287-91.

3. González Solanellasa M, Romagosa Pérez-Portabellaa A. et al. Deficiencia de vitamina D en mujeres en edad fértil. Aten Primaria. 2008;40(8):393-9.

4. Calatayud M, Jodar E, , Sánchez R, Guadalix S y Hawkins F. Prevalencia de concentraciones deficientes e insuficientes de vitamina D en una población joven y sana. Endocrinol Nutr. 2009;56(4):164-9.

5. Quesada Gómez JM., Sosa Henríquez M. Nutrición y osteoporosis. Calcio y vitamina D. Rev Osteoporos Metab Miner 2011 3; 4:165-182.

6. Ross AC, Manson JE, Abrams SA, Aloia JF, Brannon PM, Clinton SK, et al. The 2011 Report on Dietary Reference Intakes for Calcium and Vitamin D From the Institute of Medicine: What Clinicians Need to Know. J Clin Endocrinol \& Metab. 2011;96(1):53-8.

7. Sai A.J., Walters R.W, Fang X, and Gallagher J.C.. Relationship between Vitamin D, Parathyroid Hormone, and Bone Health. J Clin Endocrinol Metab, March 2011, 96(3):0000-0000.

8. Joshi D, Center JR, Eisman JA. Vitamin D deficiency in adults. Australian Prescriber. 2010;33(4):103-6.

9. Wagner CL, Greer FR, and the Section on Breastfeeding and Committee on Nutrition. Prevention of Rickets and Vitamin D Deficiency in Infants, Children, and Adolescents. Pediatrics. 2008; 122:1142-52.

10. 16 Tang BM, Eslick GD, Nowson C, Smith C, Bensoussan A. Use of calcium or calcium in combination with vitamin D supplementation to prevent fractures and bone loss in people aged 50 years and older: a meta-analysis. Lancet. 2007;370:657-66.

11. Bischoff-Ferrari HA, Dawson-Hughes B, Staehelin HB, Orav JE, Stuck AE, Theiler R, Wong JB, Egli A, Kiel DP, Henschkowski J. Fall prevention with supplemental and active forms of Vitamin D: a meta-analysis of randomised controlled trials. BMJ. 2009;339:b3692

12. Juan Pedro-Botet. Vitamina D: ¿un nuevo factor de riesgo cardiovascular? Clin InvestArterioscl.2010;22(2):72-78.

13. Vitamin D status and measures of cognitive function in healthy older European adults. Seamans, K M; Hill, T R; Scully, L; Meunier, N; Andrillo-sanchez, M; et al. European Journal of Clinical Nutrition 64.10 (Oct 2010): 1172-8.

14. Gupta D., Vashi P.G., Trukova K., Lis C.G. and Lammersfeld C.A. Prevalence of serum vitamin D deficiency and insufficiency in cancer: Review of the idemiological literature. Experimental and Therapeutic Medicine 2011 2:2 (181-193). 
15. Bjelakovic G, Gluud LL, Nikolova D, Whitfield K, Wetterslev J, Simonetti RG, Bjelakovic M, Gluud C. Vitamin D supplementation for prevention of mortality in adults. Cochrane Database of Systematic Reviews 2011, Issue 7.

16. Vitamina D. evidencias y controversias. INFAC. Volumen 20. $\mathrm{N}^{\circ} 2 \cdot 2012$. http://www.osakidetza.euskadi. net/cevime

17. Lips P, Duong T, Oleksik AM, Black D, Cummings S, Cox D, Nickelsen T, for the MORE Study Group. A global study of vitamin $\mathrm{D}$ status and parathyroid function in postmenopausal women with osteoporosis: baseline data from the multiple outcomes of raloxifene evaluation clinical trial. J Clin Endocrinol Metab 2001;86:1212-21

18. A. Catharine Ross, JoAnn E. Manson, Steven A. et all. The 2011 Report on Dietary Reference Intakes for Calcium and Vitamin D from the Institute of Medicine: What Clinicians Need to KnowJ Clin Endocrin Metab. First published ahead of print November 29, 2010 as doi:10.1210/jc.2010-2704.

19. Calvo MS, Whiting SJ, Barton CN. Vitamin D Intake: A Global Perspective of Current Status. J. Nutr. 2005;135:310-6.

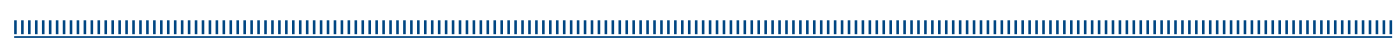

